37) Thorsen, G. and S. G. Terjesen: Chem Eng. Sci., 17, 137 (1962).

38) Ueyama, K., K. Ogawa and J. Hatanaka: J. Chem. Eng. Japan, 6, 167 (1973).

39) Vermeulen, T.: Ind. Eng. Chem., 45, 1664 (1953).

40) Ward, D. M., O. Trass and A. I. Johnson: Can. J. Chem. Eng., 40, 164 (1962).

41) Wilke, C. R. and P. Chang: AIChE J., 1, 264 (1955).

42) Winnikow, S.: Can. J. Chem. Eng., 46, 217 (1968).

43) Winnikow, S. and B. T. Chao: Phys. Fluids, 9, 50 (1966).
44) Yamaguchi, M., T. Fujimoto and T. Katayama: J. Chem. Eng. Japan, 8, 361 (1975).

45) Yamaguchi, M., T. Katayama and K. Ueyama: ibid., 7, 334 (1974).

46) Yamaguchi, M., S. Watanabe and T. Katayama: ibid., 8, 415 (1975).

(Presented at the 7th Autumn Meeting, Nagoya, Oct., (1973) and the 9th Autumn Meeting, Fukuoka, Oct., (1975) of The Soc. of Chem. Engrs., Japan.)

\title{
MASS TRANSFER TO A ROTATING SPHERE IN A STREAM
}

\author{
TAKESHI FURUTA, MORIO OKAZAKI AND RYOZO TOEI \\ Department of Chemical Engineering, Kyoto University, Kyoto 606
}

\begin{abstract}
The local and average mass transfer coefficients to a rotating sphere were measured by electrochemical method, when the angle $\alpha$ between the axis of rotation and the direction of the uniform stream were both $45^{\circ}$ and $90^{\circ}$. For measurements of the local mass transfer rates, circular isolated electrodes of $\mathbf{2} \mathrm{mm}$ dia. were used, and the time averaged values and the variations of the local mass transfer rates were obtained by processing the signals from each isolated electrode during some revolutions. Moreover, on the basis of the measurements of average mass transfer coefficients, new empirical equations were obtained with which they have been correlated well for $\alpha=0^{\circ}, 45^{\circ}$ and $90^{\circ}$.
\end{abstract}

\section{Introduction}

The study on heat and mass transfer to a rotating sphere in a stream is important in the analysis of heat or mass transfer between the circumferential fluid and the particles suspended in $i^{3}{ }^{3}$. Several studies have been reported on heat or mass transfer to/from a rotating sphere placed in a uniform stream. From a hydrodynamic point of view, these studies should be divided into two classes. When the angle $\alpha$ between the uniform stream and the axis of rotation of the sphere is equal to zero, the velocity profile around the sphere shows axially symmetry. On the other hand, when $\alpha \neq 0^{\circ}$, the velocity profile is completely three dimensional so that it becomes very difficult to study it theoretically. In the case of $\alpha=0^{\circ}$, Tanaka and $\mathrm{Tago}^{5)}$ suggested a semi-empirical equation for the average mass transfer coefficients, which could correlate the experimental results well. Also the present authors ${ }^{2)}$ obtained the local mass transfer rates by solving the laminar boundary layer equations, and the numerical results showed them in good agreement with the measurements. On the other hand, for $\alpha=$

\footnotetext{
Received December 1,1976. Correspondence concerning this article should be addressed to T. Furuta.
}

$90^{\circ}$, Noordsij and Rotte ${ }^{4)}$, and Tanaka and Tago ${ }^{51}$ measured the average Sherwood numbers, and moreover Eastop ${ }^{1)}$ obtained the average Nusselt numbers. However all these investigations have been no more than the measurements of average heat or mass transfer coefficients.

In the present work, the local mass transfer rates of a sphere rotating in a uniform stream were measured for $\alpha=90^{\circ}$ and $45^{\circ}$ by means of the so-called electrochemical method. At the same time, also the average mass transfer coefficients were obtained to provide some discussions about the effects of $\alpha$.

\section{Experimental Apparatus and Procedure}

The experimental apparatus is diagramatically shown in Fig. 1(a). Almost all the parts except for the test section are the same as that in our previous study ${ }^{2}$. The test section used for $\alpha=45^{\circ}$ is illustrated in Fig. 1(b).

In the previous investigation ${ }^{2)}$, the local mass transfer rates to an isolated electrode were stationary during a revolution of the sphere, since the velocity profile around the sphere was symmetric about the axis of rotation. However, because $\alpha$ is not zero in the present work, the velocity profile becomes three 
dimensional and then the rate of local mass transfer is considered to vary during a revolution. Thus it is necessary to determine the location of the isolated electrode during a revolution. Figure 2 show the device for this purpose. A disk is attached at the end of the rotating shaft in which a hole is drilled. A phototransister and luminous diode are set in the manner that the hole is put between them. When the disk is rotated, a pulse-called "pulse-1"-is generated during one revolution of the sphere. The location of the isolated electrode is determined by referring "pulse-1". On the rotating disk also thirty-six holes are drilled along a different circumference, which is located at the inner part of the hole for "pulse-1", to produce thirty-six pulses of the same interval per revolution. These pulses are named as "pulse-2" and used as triggering pulses to sample the $\mathrm{AD}$-converted values.

Preliminary experiments were performed in which signals from the isolated electrode (i.e. the voltage drops in a standard resister) were recorded by an electromagnetic oscillograph. The experiments, however, suggested the following two problems:

1) Since the separation point of the velocity profile around the sphere was located at about $76^{\circ}$ from the forward stagnation point*, almost all the isolated electrodes passed through the wake region during one revolution, and the mass transfer rates in this region exhibited considerable fluctuation.

2) As will be mentioned later, there existed conditions under which $S h_{l}$ became oscillating over the whole area of the sphere.

In such cases where $S h_{l}$ shows different values during every revolution, the results, which are obtained by only one record during a revolution, are not considered to be meaningful. Then in order to get useful results, it is necessary to record the signals during several revolutions and to process them statistically. In the present work, a hybrid computer equipped with AD-converters were used to process the data. Figure 3 shows the block diagram for the data processing. The signals from the isolated electrodes were amplified and recorded by a data recorder. At the same time, the two kinds of pulses mentioned above were also recorded in its other channels. After the signals were recorded during about 300 revolutions, they were reproduced and put into the computer to calculate the time averaged $S h_{l}$ and its coefficient of variation. The computer used was FACOM U-200 in Data Processing Center in Kyoto University.

The experiments were carried out at three kinds of stream Reynolds numbers for rotational Reynolds numbers between 500 and 55000. The physical prop-

\footnotetext{
* The detail about the separation point is referred to the previous investigation ${ }^{2)}$.
}

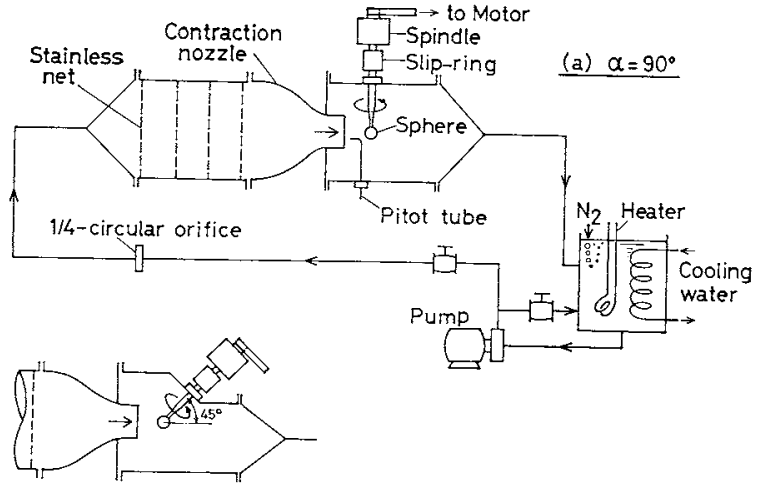

(b) $a=45^{\circ}$

Fig. 1 Schematic diagram of experimental apparatus

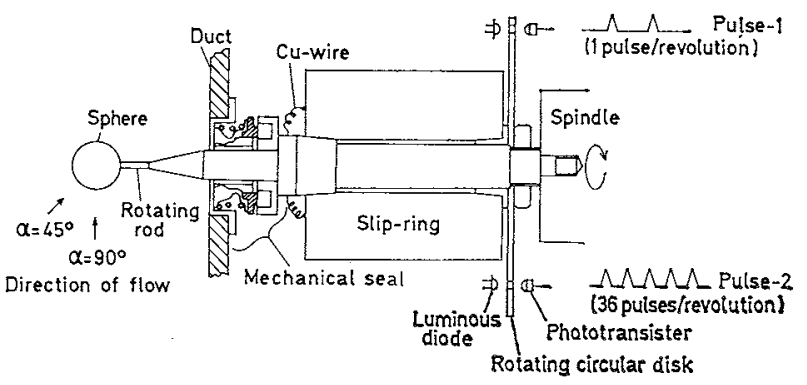

Fig. 2 Assembly of rotating sphere and devices for determination of location of isolated electrode

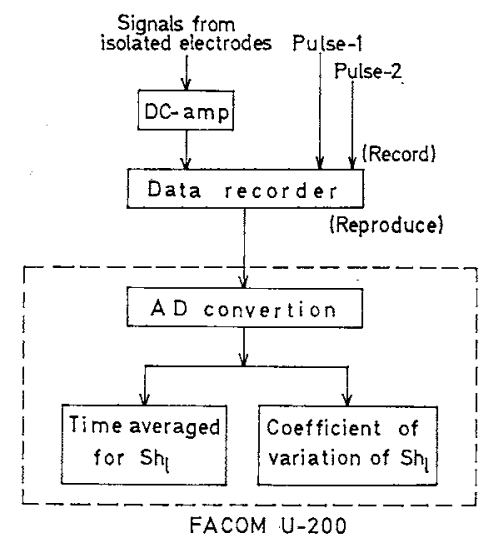

Fig. 3 Block diagram of signal processing

Table 1 Properties of the electrolytes

$\begin{array}{lll}\text { Ferricyanide conc. } & 0.005 & {[\mathrm{~mol} / l]} \\ \text { Ferrocyanide conc. } & 0.005 & {[\mathrm{~mol} / l]} \\ \text { Potassium hydroxide conc. } & 0.5 & {[\mathrm{~mol} / l]} \\ \text { Density } & 1.024 & {\left[\mathrm{~g} / \mathrm{cm}^{3}\right]} \\ \text { Viscosity } & 0.00897 & {\left[\mathrm{~g} / \mathrm{cm}^{3} \cdot \mathrm{sec}\right]} \\ \text { Diffusivity of ferricyanide ion } & 7.45 \times 10^{-6} & {\left[\mathrm{~cm}^{2} / \mathrm{sec}\right]}\end{array}$

erties of electrolyte are summarized in Table $\mathbf{1}$.

\section{Results and Discussions}

\section{1 The local Sherwood number: $S \boldsymbol{h}_{l}$}

Figures 5-10 show examples of the profile of time averaged $S h_{l}$ and their coefficients of variations, $C_{v}$. $C_{v}$ is defined as the following equation. 


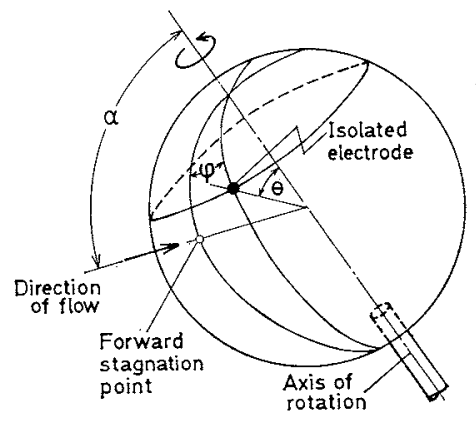

Fig. 4 Coordinate system
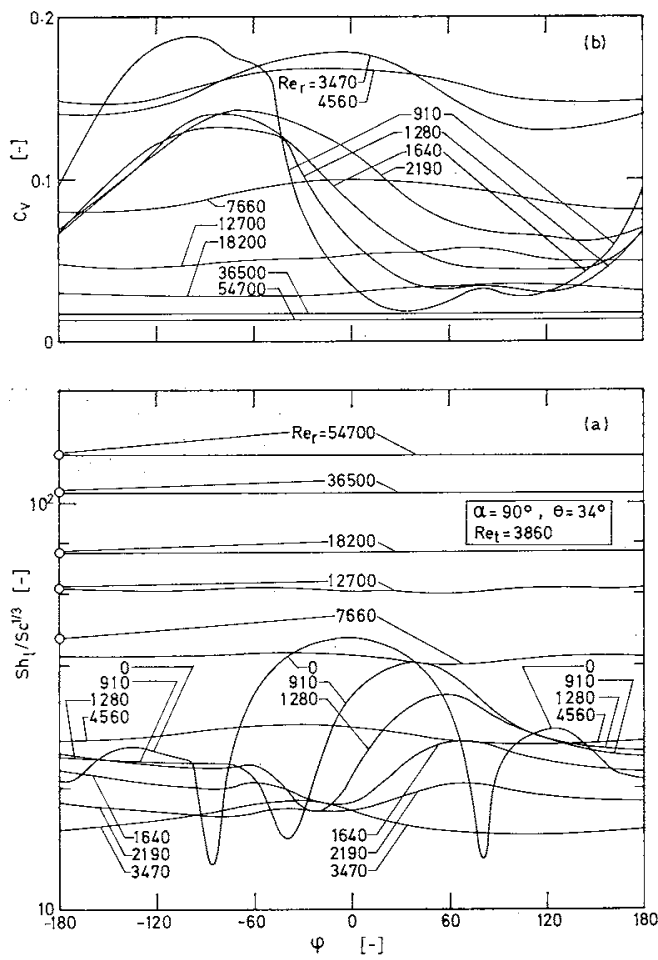

Fig. 5 Profile of $S h_{t}$ and $C_{v}$ for $a=90^{\circ}$

$$
C_{v}=\sqrt{\frac{\sum_{i}^{N}\left(S h_{l}\right)_{i}^{2}}{N}-\left(\frac{\sum_{i}^{N}\left(S h_{l}\right)_{i}}{N}\right)^{2} / \frac{\sum_{i}^{N}\left(S h_{l}\right)_{i}}{N}}
$$

where $\left(S h_{l}\right)_{i}$ is $S h_{l}$ at the $i$-th sampling (i.e. the $i$-th revolution), and the total sampling number $N$ is about 300. In the following discussions, the time averaged $S h_{l}\left(=\sum_{i}^{N}\left(S h_{l}\right)_{i} / N\right)$ is denoted for convenience as $S h_{l}$. In order to prescribe the location of the isolated electrode, two angles, $\theta$ and $\varphi$, are introduced as illustrated in Fig. 4. $\theta$ is the angle between the axis of rotation and the line connecting the isolated electrode to the center of the sphere. $\varphi$ is the circumferential angle, which is measured in the direction of rotation from the plane that contains the axis of rotation and is parallel to the free stream velocity. The total number of isolated electrodes used were nine for $\alpha=90^{\circ}$ from $\theta=0^{\circ}$ to $90^{\circ}$, and fifteen for $\alpha=45^{\circ}$
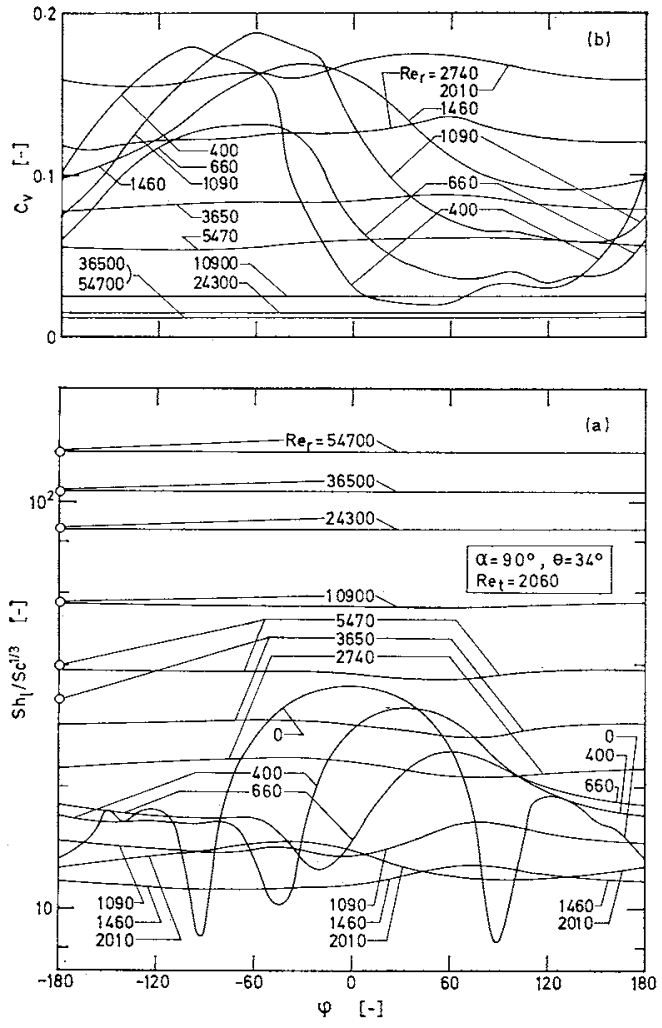

Fig. 6 Profile of $S h_{l}$ and $C_{v}$ for $\alpha=90^{\circ}$

from $\theta=0^{\circ}$ to $157^{\circ}$, which were located in approximately the same interval of $11^{\circ}$.

Figures 5-7 show the measurements for $\alpha=90^{\circ}$ and $\theta=34^{\circ}$. The isolated electrode used here passed partly the wake region during one revolution. Figures 5 (a) and (b) show the profile of $S h_{l}$ and $C_{v}$, respectively, at $R e_{t}=3860$. For comparison $S h_{l}$ in quiescent fluid are also presented on the ordinate in Fig. 5(a). At $R e_{r}=0$ (i.e. no revolution), $S h_{l}$ becomes minimum at the nearly symmetric position in each side of $\varphi$. The positions correspond to the separation points of the velocity profile over the still sphere. As the sphere begins to rotate, even if $R e_{r}$ is still very small, the separation point in the positive side of $\varphi$ vanishes. At the same time, the values of $C_{v}$ over the wake region in the positive side of $\varphi$ become gradually small as seen in Fig. 5(b). This can be interpreted as resulting from the stabilization of flow due to the revolution. On the other hand, another separation point in the negative side of $\varphi$ is progressively shifted in the positive $\varphi$-direction and the minimum value of $S h_{l}$ is gradually increased as $R e_{r}$ becomes larger. However this separation point also disappears at $R e_{r} \cong 2000$. At $R e_{r}=0, S h_{l}$ is maximum at $\varphi=0^{\circ}$ and rapidly decreases up to the separation point. As $R e_{r}$ increases this maximum point moves in the positive $\varphi$-direction and the profile of $S h_{l}$ becomes nearly flat as $R e_{r}$ becomes larger than 3470 . As shown in Fig. 5 (b), the region, where the laminar boundary layer is 

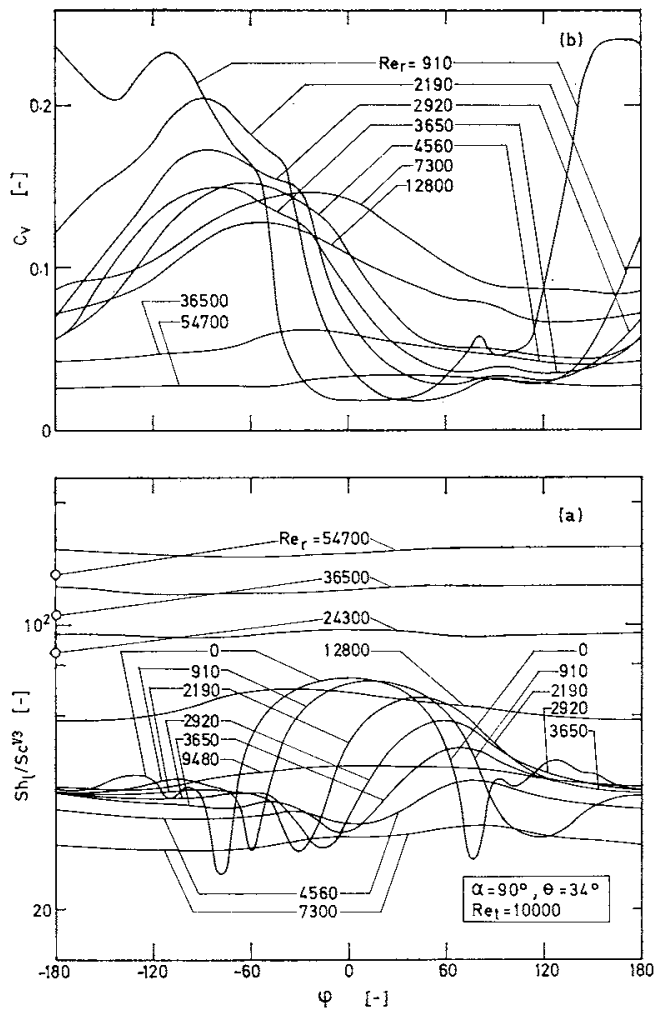

Fig. 7 Profile of $S h_{l}$ and $C_{v}$ for $\alpha=90^{\circ}$
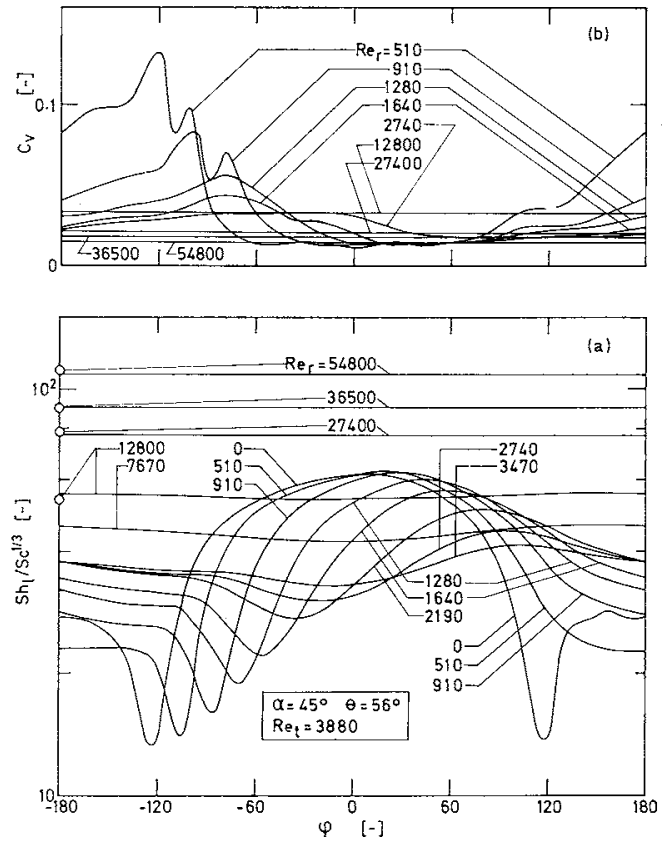

Fig. 8 Profile of $S h_{l}$ and $C_{v}$ for $\alpha=45^{\circ}$

considered to cover and then $C_{v}$ is small, is transferred to positive $\varphi$-direction and in the remaining parts $C_{v}$ grows with increase of $R e_{r}$. At $R e_{r} \cong 3470, C_{v}$ becomes large over the whole portion of $\varphi$. This is interpreted in that the large disturbances are caused as the result of the interference between the uniform stream and the velocity due to the rotation. As will be mentioned
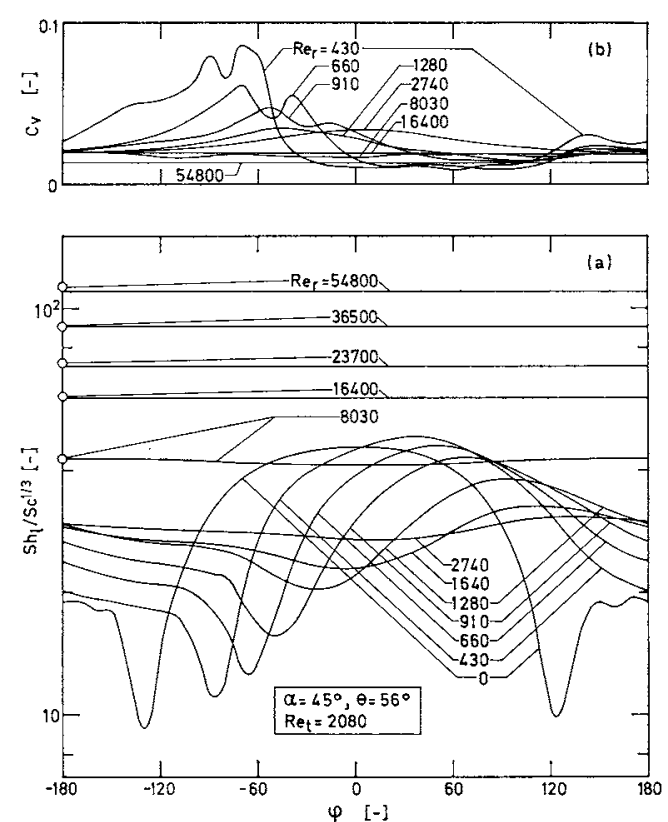

Fig. 9 Profile of $S h_{l}$ and $C_{v}$ for $a=45^{\circ}$
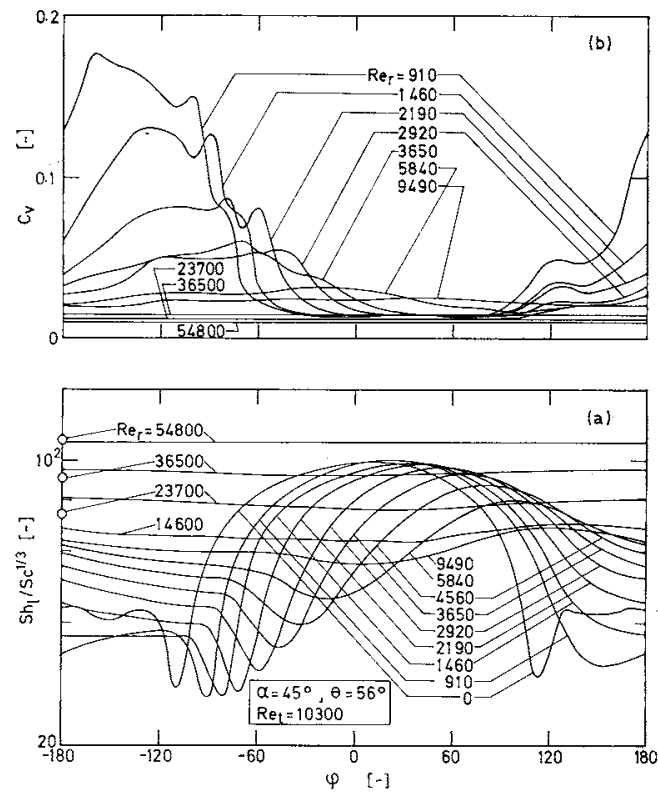

Fig. 10 Profile of $S h_{l}$ and $C_{v}$ for $\alpha=45^{\circ}$

later, this values of $\operatorname{Re}_{r}(\cong 3470)$ nearly correspond to that at which the average Sherwood number becomes minimum. When $R e_{r}$ grows larger, $S h_{l}$ increases rapidly and $C_{v}$ again decreases. This is considered due to the fact that the velocity field around the rotating sphere is approaching to the condition where the rotation is predominant. In fact, $R e_{r}$ being greater than $12700, S h_{l}$ becomes nearly equal to the value that is obtained in the quiescent fluid (i.e. $R e_{t}=0$ ). Figures 6 and 7 represent the results at $R e_{t}=2060$ and 10000 , respectively. The changes in the shape of the profile of $S h_{l}$ with $R e_{r}$ are qualitatively the same as 

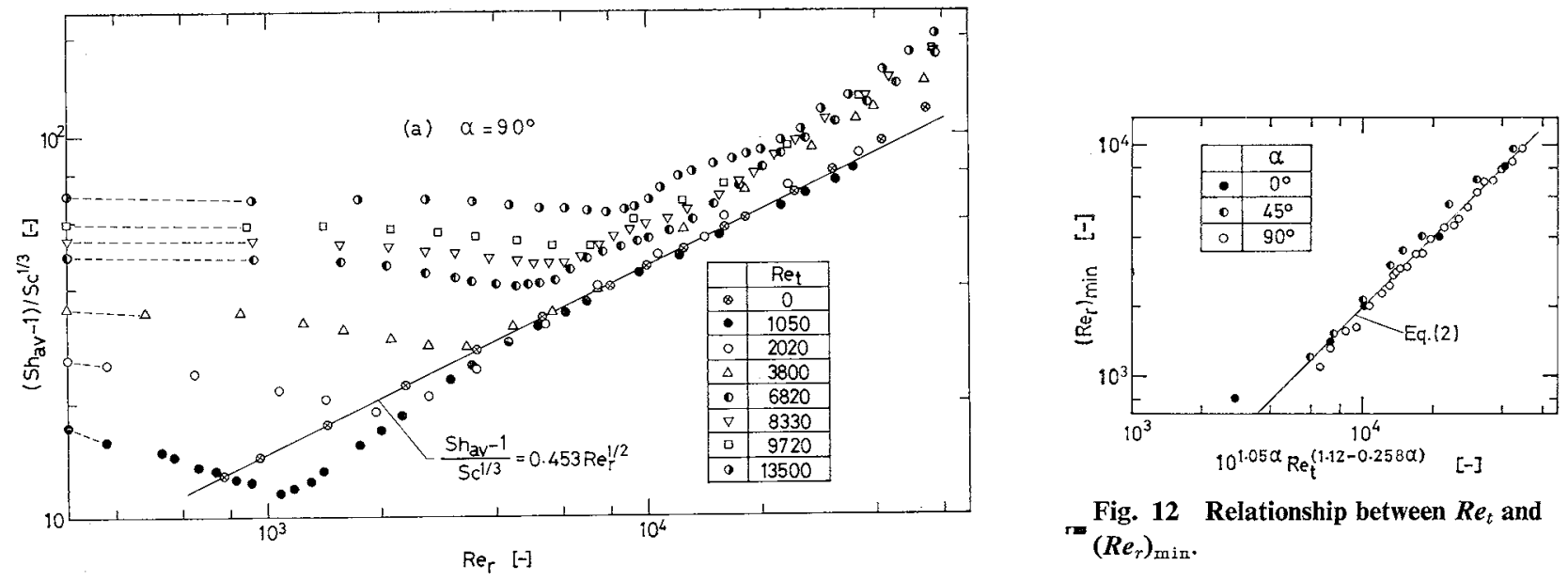

Fig. 12 Relationship between $R e_{t}$ and $\left(R e_{r}\right)_{\mathrm{min}}$.

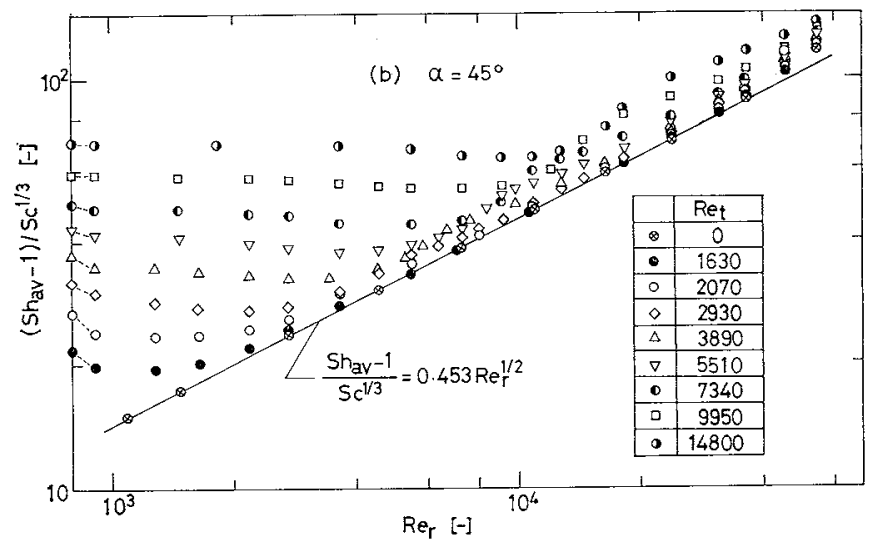

Fig. 11 Experimental results of $S h_{2 \nabla}$ of a rotating sphere for $\alpha=90^{\circ}$ and $45^{\circ}$

that in Fig. 5. However, the displacement rate of the maximum point of $S h_{l}$ is slower as $R e_{t}$ increases and the lower limit at which $S h_{l}$ matches the value for $R e_{t}=0$ becomes larger. Especially at $R e_{t}=10000$, within the limit of the present experiment, $S h_{l}$ does not completely coincide with the $S h_{l}$ at $R e_{t}=0$.

Figures 8-10 show the results for $\alpha=45^{\circ}$ and $\theta=56^{\circ}$ at $R e_{t}=3880,2080$ and 10300 , respectively. The whole changes of profile of $S h_{l}$ with $R e_{r}$ are almost similar to that for $\alpha=90^{\circ}$. However, $C_{v}$ decreases monotonously over the whole area of the sphere as $R e_{r}$ increases.

The experimental results of $S h_{l}$ obtained by each isolated electrodes are integrated over the whole area of the sphere to calculate the average Sherwood numbers. The calculated values are in good agreement with that measured within the accuracy of $\pm 5 \%$ at various $R e_{t}$ and $R e_{r}$ for both $\alpha=90^{\circ}$ and $45^{\circ}$.

\section{2 The average Sherwood number: $S h_{\mathrm{av}}$}

The measurements on $S h_{\mathrm{ar}}$ for $\alpha=90^{\circ}$ and $45^{\circ}$ are

\footnotetext{
* When $\alpha=0^{\circ}, S h_{\mathrm{av}}$ shows no minimum value. So that in this case $\left(R e_{r}\right)_{\min }$ is defined as $R e_{r}$ at which $S h_{\mathrm{a} v}$ becomes larger than that for $R e_{r}=0$.
}

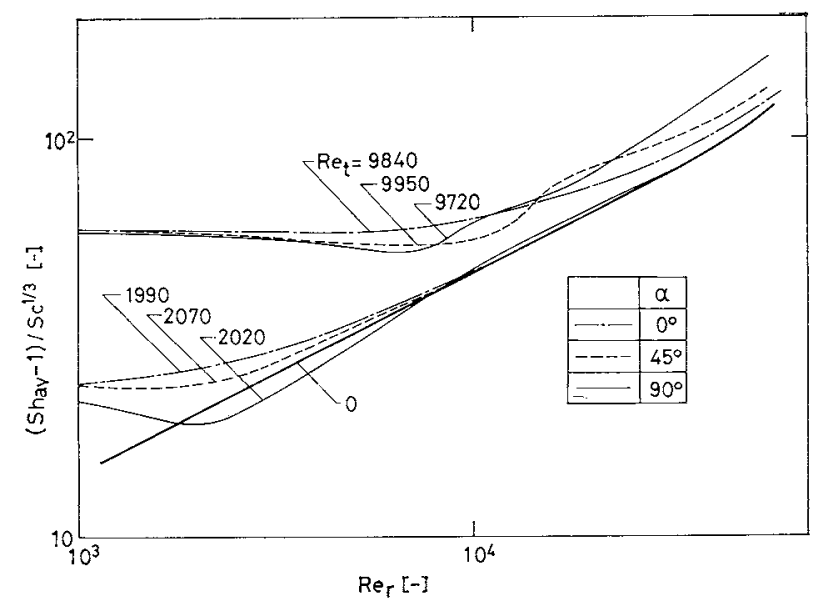

Fig. 13 Effect of $\alpha$ on $S h_{\mathrm{av}}$

shown in Figs. 11(a) and (b), respectively. In the figures, the data plotted on the ordinate refer to $S h_{a, v}$ at $R e_{r}=0$. Here $S h_{\mathrm{ar}}$ reduces gradually as $R e_{r}$ increases at constant $R e_{t}$. After being minimum, $S h_{\mathrm{av}}$ grows again. The ratio of reduction of $S h_{\mathrm{av}}$ is generally larger as $R e_{t}$ is small, and for the same $R e_{t}$ the ratio is slightly larger for $\alpha=90^{\circ}$ than that for $\alpha=45^{\circ}$. Let $R e_{r}$ at which $S h_{\mathrm{av}}$ becomes minimum expresses as $\left(R e_{r}\right)_{\min }{ }^{*}$. The relationship between $\left(R e_{r}\right)_{\min }$ and $R e_{t}$ is shown in Fig. 12 and is represented as the following equation.

$$
\begin{gathered}
\left(R e_{r}\right)_{\min }=0.195 \times 10^{1.05 \alpha} R e_{t}^{(1.12-0.258 \alpha)} \\
(\alpha: \text { radian })
\end{gathered}
$$

When $R e_{t}$ is about $3800,\left(R e_{r}\right)_{\min }$ is about 3500 and 3200 for $\alpha=90^{\circ}$ and $45^{\circ}$, respectively, which nearly coincide with $R e_{r}$ where $S h_{l}$ becomes minimum. As pointed out by Tanaka and Tago $^{5)}$ and in our previous work, ${ }^{2)} S h_{\mathrm{av}}$ for $\alpha=0^{\circ}$ showed no tendency to decrease as $R e_{r}$ increased. The systematic comparison of $S h_{\mathrm{av}}$ for these three cases of $\alpha$ is illustrated in Fig. 13 at $R e_{t} \cong 2000$ and 10000 . It should be noted that for $\alpha=90^{\circ}$ and $R e_{t}=2000, S h_{\mathrm{av}}$ becomes smaller near $\left(R e_{r}\right)_{\text {min }}$ than that at $R e_{t}=0$. This phenomenon has 
been indicated at first by Noordsij and Rotte ${ }^{4)}$. For $\alpha=90^{\circ}$ and at large $R e_{t}, S h_{\mathrm{ar}}$ cannot coincide with the value for $R e_{t}=0$ even at large $R e_{r}$ within the limit of this study.

Tanaka and Tago $^{5)}$ suggested following semiempirical equation to correlate $S h_{\mathrm{av}}$ for $\alpha=0^{\circ}$ and $90^{\circ}$.

$$
\begin{aligned}
\left(S h_{a v}-1\right) / S C^{1 / 3} R e_{t}^{0.5}= & \left\{\left|A \pm B\left(a \omega / U_{\infty}\right)^{1.5}\right|\right\}^{1 / 3} \\
& \left(+: \alpha=0^{\circ},-: \alpha=90^{\circ}\right)
\end{aligned}
$$

where the constants $A$ and $B$ are obtained from the empirical equations of $S h_{\mathrm{a} v}$ for $R e_{r}=0$ and $R e_{t}=0$, respectively.

In the present experiments $A=0.185$ and $B=0.093$ in contrast to $A=0.0589$ and $B=0.0166$ obtained by Tanaka and Tago $^{5) *}$. As mentioned in our previous work ${ }^{2}$, Eq. (3) could correlate the experimental results for $\alpha=0^{\circ}$ satisfactorily. The comparison between the experimental results for $\alpha=90^{\circ}$ and that calculated by Eq. (3) is represented in Fig. 14. The values of $a \omega / U_{\infty}$ where $\left(S h_{\mathrm{av}}-1\right) / S c^{1 / 3} R e_{t}^{1 / 2}$ becomes minimum are quite different between the measurements and the calculations under the various conditions, especially for large $R e_{t}$. It seems difficult to express the experimental results satisfactorily by Eq. (3). It is clear from Figs. 11(a) and (b) that the curves joining $S h_{\mathrm{a} v}$ at each $R e_{t}$ are nearly similar to one another. Then these curves would be fitted by some adequate transpositions. The following equation is empirical one under such consideration.

$$
\begin{aligned}
\frac{S h_{\mathrm{av}}-1}{S c^{\mathrm{1} / 3} R e_{t}^{(0.67-0.0388 \alpha)}=} & \left(0.088+0.049 \alpha-0.00242 \alpha^{2}\right) \\
& \times\left\{R e_{r} /\left(R e_{r}\right)_{\min }\right\}^{(0.48+0.0675 \alpha)} \\
(\text { for } 1.0 & \left.\leqq R e_{r} /\left(R e_{r}\right)_{\min } \leqq 60\right) \\
S h_{\mathrm{av}}-1 & \left(0.58-0.45 \alpha+0.151 \alpha^{2}\right) \\
S c^{1 / 3} R e_{t}^{(0.5+0.07 \alpha)}= & \times\left\{R e_{r} /\left(R e_{r}\right)_{\min }\right\}^{-0.051 \alpha}
\end{aligned}
$$$$
\text { (for } \left.0.1 \leqq R e_{r} /\left(R e_{r}\right)_{\min } \leqq 1.0\right)
$$

where $\alpha$ is represented as radian in these equations. The comparisons between the measurements and the calculations by Eqs. (4) and (5) are shown in Figs. 15 (a) to (c). As a whole, the measurements are correlated well with Eqs. (4) and (5). However, especially for $\alpha=0^{\circ}$ and $45^{\circ}$, there are some scattering in the range of $R e_{r} /\left(R e_{r}\right)_{\min }$ between 1.0 and 3.0.

The theoretical analysis on the local mass transfer coefficient of the rotating sphere is a problem for a future paper.

\section{Conclusion}

The local Sherwood numbers $S h_{l}$ during a revolution of a rotating sphere in a uniform stream were measured for $\alpha=45^{\circ}$ and $90^{\circ}$ by means of electro-

* The detail discussion about the constants is referred to in the previous work ${ }^{2)}$.

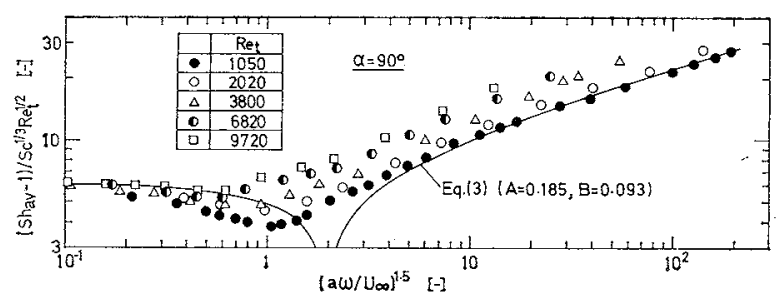

Fig. 14 Comparison between experimental results of $S h_{\mathfrak{a v}}$ and Tanaka's equation
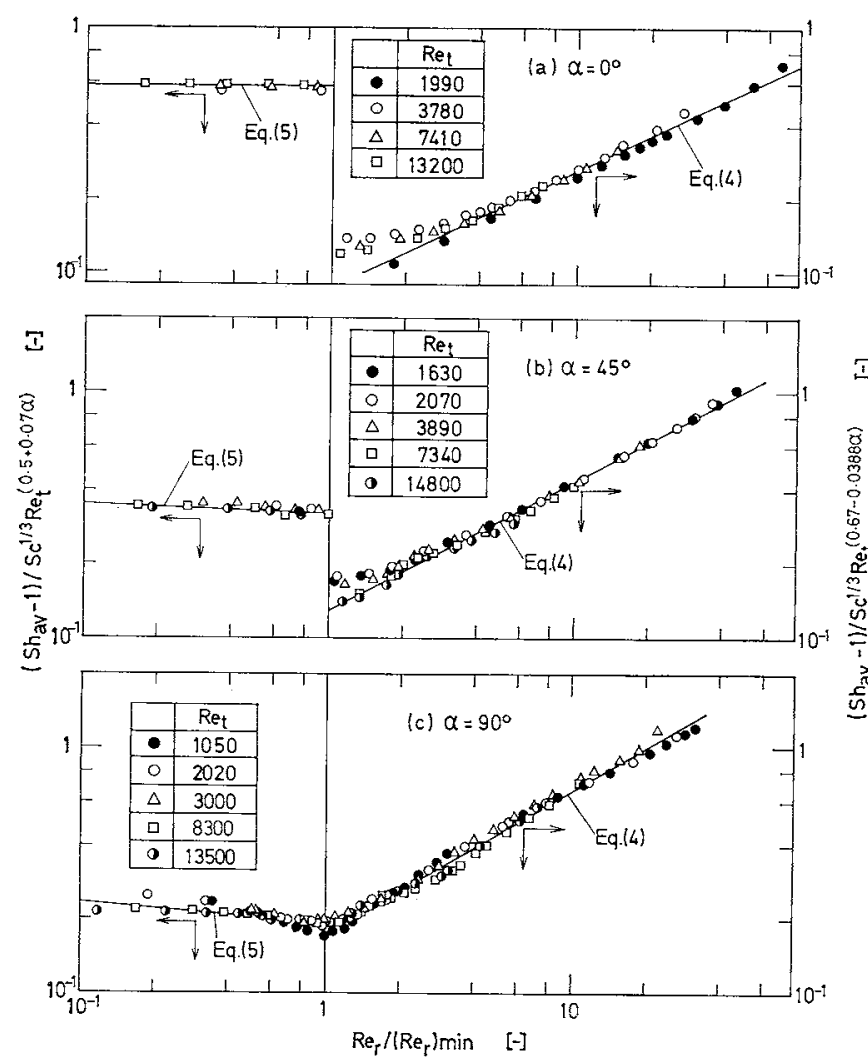

Fig. 15 Comparison between experimental results of $S h_{\mathrm{\varepsilon} \vee}$ and Eqs. (4) and (5)

chemical method using circular isolated electrodes. As $R e_{r}$ increases, the shape of the profile of $S h_{l}$ changes from that in stream predominant region to that in rotation predominant one, and at the boundary between them $S h_{l}$ fluctuates violently.

$S h_{l}$ for each isolated electrode was integrated over the whole area of the rotating sphere to calculate the average values. The calculations were in good agreement with $S h_{\mathrm{ar}}$ measured within an accuracy of $\pm 5 \%$ under various conditions.

New empirical equations for $S h_{\mathrm{av}}$, Eqs. (4) and (5), were suggested for various $\alpha$ between $0^{\circ}$ and $90^{\circ}$.

\section{Acknowledgment}

The authers thank Mr. N. Fujii for his technical assistance.

\section{Nomenclature}

$\begin{array}{lll}a & =\text { radius of sphere } & \text { [cm] }\end{array}$

$C_{v} \quad=$ coefficient of variation $\quad[-]$ 


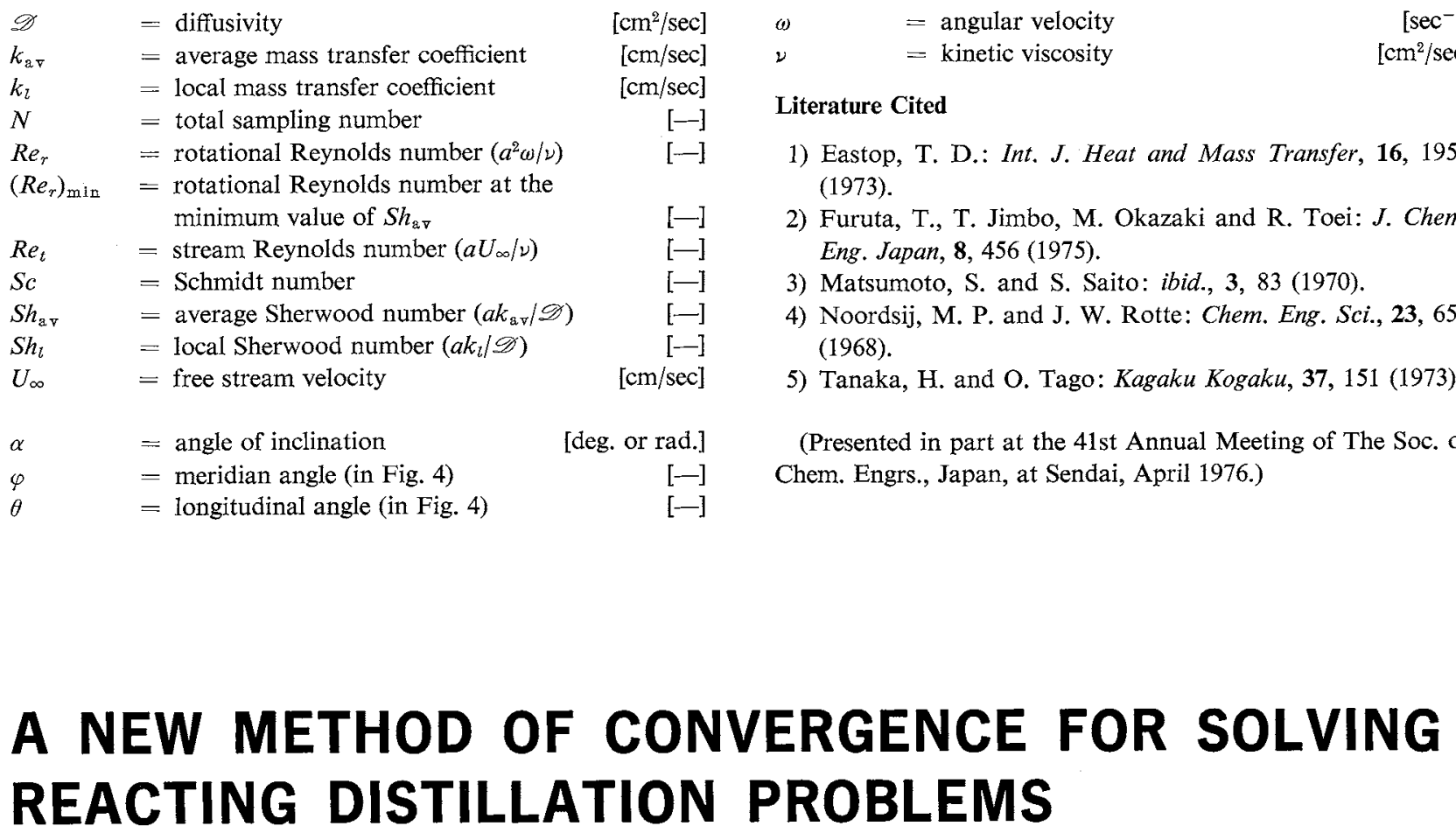

\author{
Hiromasa KOMATSU and Charles D. HOLLAND \\ Department of Chemical Engineering, Texas $A \& M$ University, \\ College Station, Texas 77843
}

\begin{abstract}
A new correction factor $(\eta)$ for reaction rate has been defined. It is analogous to $\theta$ for correcting molal flow rates in the multi- $\theta$ method of convergence. Then a new calculation method with two such kinds of the independent variables as $\theta$ and $\eta$ has been developed for solving reacting distillation problems at the steady state and at the unsteady state.

Convergence is obtained without difficulty in the case where vapor liquid equilibrium ratios may be formulated as a function of temperature only. However, in the case of calculations based on experimental data in the esterification systems, the values of $\Delta \eta$ and $\Delta \theta$ resulted from the Newton Raphson's method have some time a large number. Therefore the correction method for these by use of arctangent function has been proposed in order to avoid divergence.

This new calculation method is called "Multi $\theta$ - $\eta$ method of convergence".
\end{abstract}

\section{Introduction}

The multi $\theta$ method of convergence has been proposed for solving multi stage distillation problems at steady state and at unsteady state by Holland ${ }^{4-8)}$.

This method has been developed by use of a new correction factor $(\eta)$ for the reaction rate, $\eta$ is analogous to $\theta$ for correcting the molal flow rate in the multi $\theta$ method of convergence. A new method with such two kinds of independent variables as $\theta$ and $\eta$ has been developed for solving reacting distillation problems.

This method is called the "Multi $\theta-\eta$ method of convergence".

This method is not only useful for steady state

Received June 28,1976 . Correspondence concerning this article should be addressed to H. Komatsu, Numazu College of Technology, Numazu 410. problems, but also it is applicable for unsteady state problems by use of the implicit method.

\section{Calculation Fundamentals}

Specifications for the molal flow rate of distillate, waste and over-flow, the molal holdup of liquid, and liquid compositions are given by the multiplier $\theta_{j}$ as shown in Eq. (1) through Eq. (5)

$$
\begin{aligned}
& B_{c o}=\theta_{-1}(B / D)_{a} D_{c o} \\
& b_{i c o}=\theta_{-1}\left(b_{i} / d_{i}\right)_{a} d_{i c o} \\
& u_{j i c o}=\theta_{j}\left(u_{j i} / d_{i}\right)_{a} d_{i c o} \\
& U_{j c o}=\sum_{i=1}^{c} u_{j i c o} \\
& x_{j i}=\left(u_{j i} / U_{j}\right)_{c o}, \quad y_{j i}=K_{j i}\left(u_{j i} / U_{j}\right)_{c o}
\end{aligned}
$$

In order to derive the component material balance for the whole tower, the holdup of the vapor on each 